\title{
Surgical management of Gorham-Stout syndrome involving the cervical spine with bilateral pleural effusion: A case report and literature review
}

\author{
KE-JIE CHANG ${ }^{*}$, MENG-HANG YANG ${ }^{*}$, BING LI and HAI HUANG \\ Department of Respiratory and Critical Care Medicine, Changzheng Hospital, \\ Second Military Medical University, Shanghai 200003, P.R. China
}

Received April 18, 2019; Accepted December 3, 2019

DOI: $10.3892 /$ etm.2020.8627

\begin{abstract}
Gorham-Stout syndrome (GSS) is a rare disease characterized by spontaneous and progressive osteolysis caused by benign proliferation of lymphatic vessels or capillaries. It most commonly occurs in children or young individuals without any inherited predisposition. GSS most commonly affects the shoulder girdle, pelvis, ribs and skull. Its diagnosis is mainly based on radiological and pathological findings. The present study reports on the case of a 22-year-old male patient diagnosed with GSS involving the C1-T1 vertebrae accompanied by bilateral pleural effusion. Resection of the occipital and cervical vertebral lesions and spinal reconstruction using an internal fixator were successfully performed via the posterior approach. After the surgery, the patient received bisphosphonate treatment and vitamin D supplementation. The pleural effusion gradually decreased. At the 18-month follow-up visit, no evidence of new bone obstruction was present and the patient had no neurological sequelae.
\end{abstract}

\section{Introduction}

Gorham-Stout syndrome (GSS), also named disappearing bone disease, phantom bone disease or massive osteolysis, is a rare osteolytic disease resulting from benign lymphovascular proliferation (1). It was first described as 'the boneless arm' in 1838 (2). Since then, only 300 cases have been reported worldwide. GSS may involve any bone of the body and most

Correspondence to: Professor Hai Huang or Professor Bing Li, Department of Respiratory and Critical Care Medicine, Changzheng Hospital, Second Military Medical University, 415 Fengyang Road, Shanghai 200003, P.R. China

E-mail: haihuang7207@smmu.edu.cn

E-mail: libing1962@smmu.edu.cn

${ }^{*}$ Contributed equally

Key words: Gorham-Stout syndrome, cervical spine, pleural effusion, surgical management commonly affects the shoulder girdle, pelvis, ribs and skull. Owing to its rarity and non-specific clinical manifestation, accurate diagnosis is difficult and usually delayed. The conditions of certain patients can progress to complete bone destruction, while those of other patients are self-limiting. Patients with GSS complicated with spinal involvement or chylothorax usually have poor prognosis (3). The treatment options, including surgery, radiotherapy and medical therapy, remain controversial. Stable spinal lesions may be treated with conservative treatments, including braces, halo tractions, radiotherapy and medical therapy (4). To maintain stability and prevent neurological injury, surgical management is required for unstable spinal lesions (5). The present study reports on a case of GSS in a 22-year-old male involving the C1-T1 vertebrae with bilateral pleural effusion and discusses the diagnostic characteristics and therapeutic management.

\section{Case report}

In July 2017, a 22-year-old male was admitted to a local hospital with neck pain and limited movement of the neck for 1 month. The relevant medical history of the patient's family was uneventful and the patient had no history of trauma. A CT scan revealed multiple osteolysis of the occipital bone and several cervical vertebrae and thoracic vertebrae with a mildly increased fluorodeoxyglucose uptake accompanied by an adjacent soft tissue mass. A needle biopsy of the cervical vertebrae was performed and the results revealed hyperplasia of the fibrous tissues in the bone lesions without evidence of malignancy. No definite diagnosis was made at that time. Considering the complexity and high risk associated with surgery, the patient underwent irradiation treatment of the cervical vertebrae once at the local hospital.

At 1 week following radiation treatment, the patient developed shortness of breath and chest tightness. Chest ultrasound demonstrated large bilateral pleural effusion. The patient then presented at the Department of Respiratory and Critical Care Medicine, Changzheng Hospital (Shanghai, China). On examination, the patient was conscious but distressed and his neck was immobilized using a cervical gear. The patient had percussion dullness, decreased vocal resonance and diminished breath sounds bilaterally, but no palpable lymph 
nodes, edema of the lower extremities or neurological deficits. Laboratory examination, including routine blood tests, biochemical tests and serum tumor markers, were normal, except for albumin levels of $25 \mathrm{~g} / 1$ (normal range based on observations at Changzheng Hospital, 40-55 g/l). Radiography revealed marked osteolysis in the occipital bone, as well as C2-7 and T2 vertebrae (Fig. 1). On MRI, low signal intensity in the occipital bone and C1-T1 vertebrae was observed in the T1-weighted images (Fig. 2). The corresponding T2-weighted MRI revealed an inhomogeneous high signal intensity within the lesions (Fig. 2). The chest CT scan revealed a large amount of bilateral pleural effusion (Fig. 3). A chest catheter was inserted to the right pleural cavity for daily drainage and bloody pleural fluid was collected for further analysis. To determine the cause of the bloody pleural effusion, left-sided thoracoscopy was performed, but no abnormalities were identified. Approximately 11 of bloody pleural fluid was drained from the left pleural cavity during surgery. Pleural effusion samples were gathered from the patient on the 1st, 2nd and 3rd day post-surgery. Cytological analysis of the pleural fluid was performed and the results were negative regarding malignancies. The patient exhibited a rapid accumulation of pleural fluid within 5 days after thoracoscopy and pleural aspiration yielded a milky white fluid, which was proven to be chyle via laboratory analysis. The patient was provided with a low-fat diet and received intravenous albumin infusion.

Considering the spinal instability caused by extensive vertebral involvement and the possibility of pathological fracture, the patient was then transferred to the Department of Osseous Oncology at Changzheng Hospital for surgical intervention. The surgery was performed via a posterior approach. During the surgery, it was observed that the spinous processes of the C2-7 vertebrae had almost disappeared and had manifested as malacia, the blood supply was highly abundant, multiple vertebral plates and occipital protuberances were destroyed and chyle outflowed from the bone lesions. Pedicle screws were then inserted into the pedicles of the bilateral T3, T1 and C2 vertebrae, left-side T2 and C5 vertebrae and right-side T3 and $\mathrm{T} 7$ vertebrae. The remaining pedicle bones could not be treated in the same way, as they were almost destroyed. The occipital protuberance was largely destroyed and the cerebral dura mater was visible; therefore, the damaged bone and soft tissues were resected and a screw and an internal fixator were installed in the undamaged occipital cortex. A connecting rod was fixed to the occipital and bilateral pedicle screws. To relieve the pressure at the dural sac, the soft tissues behind the C2-7 vertebrae was carefully separated and removed. The spinous processes and vertebral plates of the T1-3 vertebrae were resected, the damaged bone was scraped off and a gelatin sponge was inserted into the site of damage of the T1-3 vertebrae. Histopathological examination of the excised tissues revealed proliferative fibrous tissues and numerous thin-walled blood vessels in the affected bone cavity, without evidence of cellular atypia and osteogenesis reactions (Fig. 4).

Based on the clinical, radiological and pathological results, the patient was eventually diagnosed with GSS. The surgery was effective and no surgery-associated complications were observed (Fig. 5). Thereafter, the patient received $4 \mathrm{mg}$ of zoledronic acid by intravenous administration every 2 months. He was also provided with calcium and vitamin D supplementation. The pleural effusions gradually decreased after surgery and the patient was discharged from our hospital at 10 days post-surgery without any neurological complications. At the 18-month follow-up visit, there was no evidence of new bone resorption.

\section{Discussion}

GSS is a rare osteolytic disease, which has received substantial attention since it was first described in 1838 (6). This disease has no features of inheritance and may occur at any age, but frequently affects children or young individuals (7).

The etiology and pathogenesis of GSS remain poorly understood. Gorham and Stout (6) speculated that abnormal hyperplasia of vascular tissues in the bone marrow cavity results in mechanical pressure, local hypoxia and acidosis, thus leading to bone resorption. Osteolysis was also thought to correlate with the increased number or enhanced activity of osteoclasts in bone lesions (8). Various studies have suggested that perivascular monocytes are involved in bone resorption $(9,10)$. Furthermore, the serum levels of basic fibroblast growth factor, vascular endothelial growth factor (VEGF)-A, VEGF-C and interleukin-8 were found to be markedly elevated in certain patients, indicating their potential roles in GSS (11,12).

In 1983, Heffez et al (13) put forward 8 diagnostic criteria for GSS: i) Positive biopsy for angiomatous tissue; ii) absence of cellular atypia; iii) minimal or no osteoblastic response and absence of dystrophic calcification; iv) evidence of local and progressive osseous resorption; v) non-expansile and non-ulcerative lesion; vi) absence of visceral involvement; vi) osteolytic radiographic pattern; viii) negative hereditary, metabolic, neoplastic, immunologic or infectious etiology. In the present case, the radiographical and CT images indicated multiple sites of osteolysis of the occipital bone and multiple cervical vertebrae, and the patient was initially suspected to have primary malignant or metastatic bone tumors. However, extensive workup, including laboratory examination, systemic CT scan, thoracoscopy and needle biopsy of the cervical vertebra did not provide any evidence of neoplastic, endocrinological or infectious diseases. To obtain an accurate diagnosis and receive further treatment, the patient was transferred to the Department of Osseous Oncology at Changzheng Hospital for surgical intervention. Post-operative histopathological examination of the excised occipital and cervical lesions revealed that the bone cavity was infiltrated with numerous dilated blood vessels and fibrous tissues without cellular atypia; thus, the patient was eventually diagnosed with GSS.

Owing to its rarity, no standard treatment is available for GSS. The treatment options include surgery, radiation therapy and medical therapy, depending on the location and size. The morbidity and mortality are high when the cervical spine is involved, as cervical spinal cord injury or cerebrovascular accident may occur. Between 1997 and 2019, only 17 cases affecting the cervical spine (including the present case) have been reported in the English literature. Details of such cases are summarized in Table I (14-25). Surgical treatment was performed in 9 patients (52\%), of whom 2 patients also received radiotherapy. The most common surgical method is spinal fusion using an internal fixator, e.g. a screw and a plate, or 


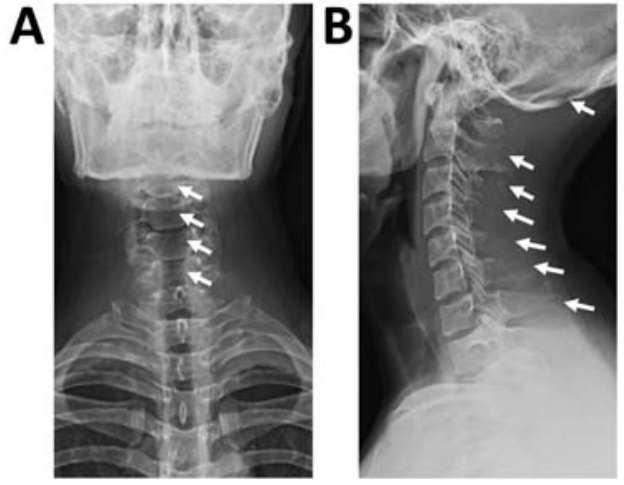

Figure 1. (A) Anteroposterior and (B) lateral images of the cervical vertebrae indicate marked osteolysis within the occipital bone and C2-7 and T2 vertebrae (arrows).
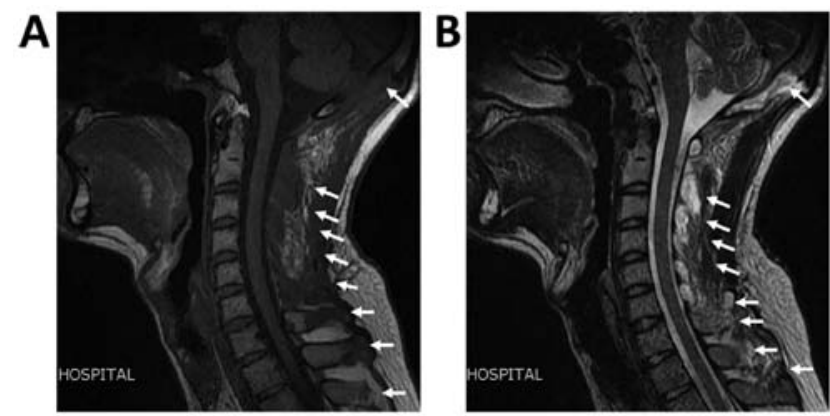

Figure 2. MRI of the cervical vertebrae. (A) Low signal intensity was observed on the T1-weighted image and (B) an inhomogeneous high signal intensity was present on the T2-weighted image within the occipital bone, C1-7 and T1 vertebrae, as well as the surrounding soft tissues (arrows).
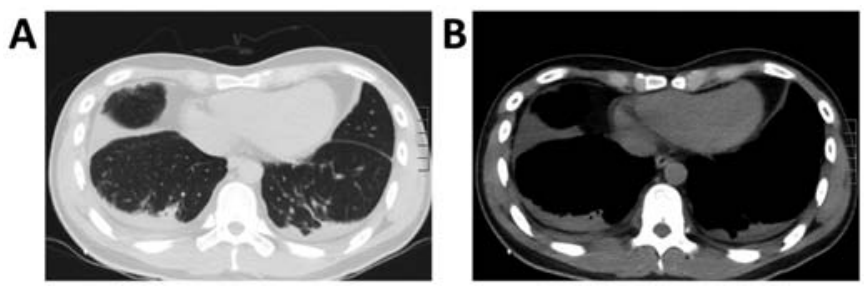

Figure 3. Chest CT scan revealing bilateral pleural effusions in (A) the lung window and (B) mediastinal window.

bone graft reconstruction. The anterior or posterior approach may be selected according to the specific condition. Of these 9 reported cases, 2 had no description of the disease progression during follow-up. Osteolysis stopped progressing in 6 of the other 7 cases. Only 1 case developed more severe osteolysis during follow-up. The majority of cases have favorable outcomes when adequate spinal fusion is performed. Radiotherapy on its own or combined with medication was administered to 8 patients (47.2\%). The outcomes of patients who receive radiotherapy may be different. Among these previous cases, 2 patients had new bone formation, 1 patient exhibited no further bone resorption, 3 patients had progressive osteolysis and 2 patients died.

In the case of the present study, remediation of dangerous cervical vertebral instability and prevention of spinal cord injury were accomplished via posterior resection of the occipital and cervical vertebral lesions and spinal reconstruction using an
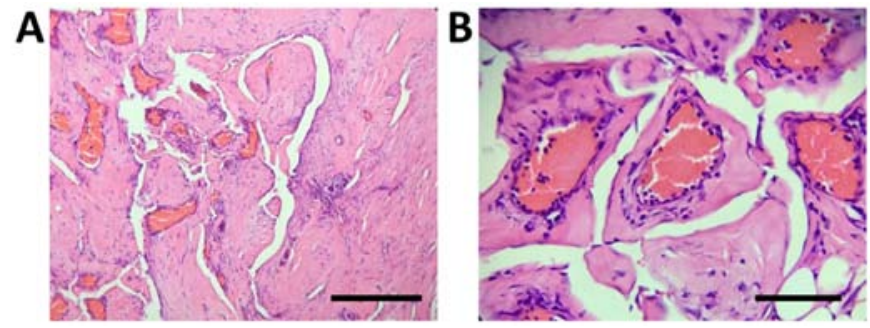

Figure 4. Histological images of the excised cervical vertebrae indicate proliferative fibrous tissues and numerous thin-walled blood vessels in the bone cavity without evidence of cellular atypia and osteogenesis reactions. (A) Magnification, x100, scale bar, $200 \mu \mathrm{m}$; (B) magnification, x400, scale bar, of $50 \mu \mathrm{m}$; Hematoxylin and eosin staining.
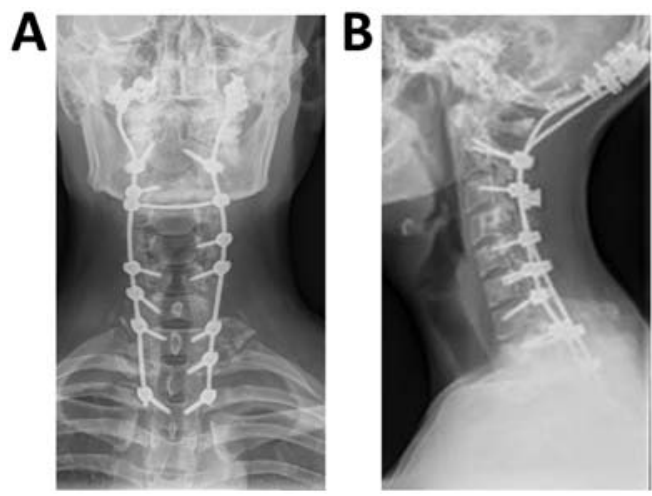

Figure 5. Post-operative (A) anteroposterior and (B) lateral plain radiographs of the cervical vertebrae indicate an internal fixator within the occipital bone, several cervical vertebrae and $\mathrm{T} 3$ vertebrae.

internal fixator. As the C1-T1 vertebrae were involved, the surgery was fairly difficult but successful and no post-operative complications occurred. Biphosphonates are a potent inhibitor of osteoclasts and may suppress bone resorption in GSS (26). Therefore, the patient was administered $4 \mathrm{mg}$ zoledronic acid every 2 months and vitamin D supplements post-surgery. At the time of conclusion of the study, the patient had no procedure-associated complications and osteolysis had not progressed.

Chylous effusions usually occur in patients with GSS with rib, clavicular, sternal, scapular or vertebral involvement (7). Chylothorax may result from direct pleural involvement or obstruction of the thoracic duct by lymphovascular tissues (27). Bilateral chylothorax is a clinically challenging condition and has a high mortality rate of 53\% (28). Common interventions include thoracic duct ligation, pleurodesis, pleurectomy, resection of lymphangiomatous lesions, radiotherapy and medical therapy (28). In the present case, the patient was provided with a low-fat diet and received intravenous albumin infusion and chest catheter drainage. Chyle outflowing from the bone lesions was observed during surgery and the lymphangiomatous tissues were surgically resected from the affected bones. The pleural effusion gradually decreased after surgery.

In conclusion, the present study reported on a case of GSS extensively invading the C1-T1 vertebrae accompanied by a large amount of bilateral pleural effusion. This case exemplifies that when osteolysis of unknown causes occurs, GSS, although rare, should be taken into consideration. Bloody or chylous pleural effusions may occur in patients with GSS with pleural involvement 


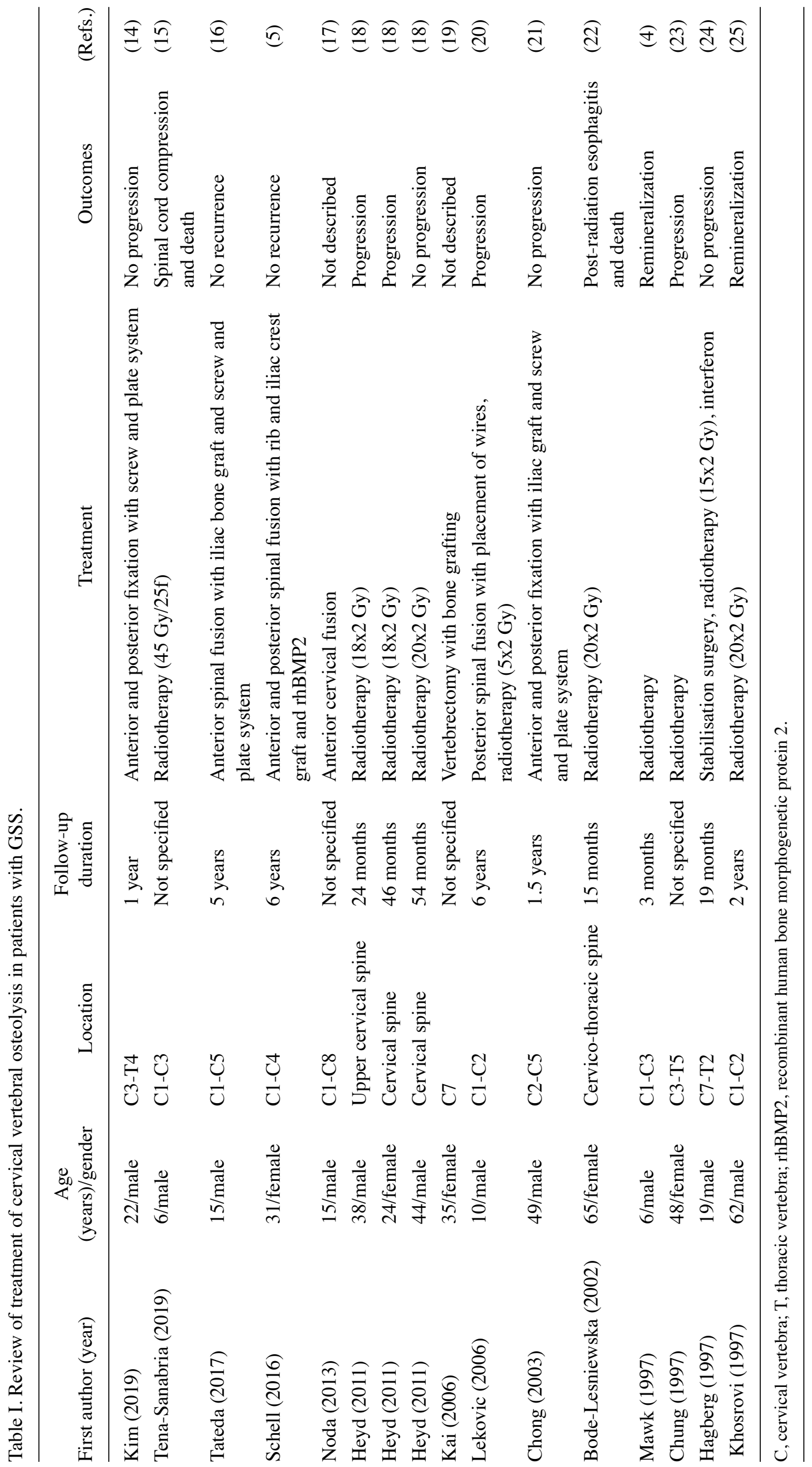


or thoracic duct obstruction. For patients with cervical spinal involvement, rapid surgical interventions are recommended to achieve spinal stability and prevent neurological complications. In the present case, the clinical outcome was favorable after surgical intervention and bisphosphonate treatment.

\section{Acknowledgements}

Not applicable.

\section{Funding}

This study was supported by the National Natural Science Foundation of China (grant nos. 81602618 and 81672929).

\section{Availability of data and materials}

The datasets used and/or analyzed during the current study are available from the corresponding author on reasonable request.

\section{Authors' contributions}

KJC interpreted the data and prepared the manuscript. MHY prepared the discussion section including the literature search and analyzed the data of previously reported cases.HH and BL performed the data acquisition and revised the final draft. All authors read and approved the final manuscript.

\section{Ethics approval and consent to participate}

Written informed consent was acquired from the patient prior to participation in this study. The present study was approved by the Ethics Committee of Shanghai Changzheng Hospital.

\section{Patient consent for publication}

Written consent was provided by the patient for the publication of his data and images.

\section{Competing interests}

The authors declare that they have no competing interests.

\section{References}

1. Dickson GR, Hamilton A, Hayes D, Carr KE, Davis R and Mollan RA: An investigation of vanishing bone disease. Bone 11: 205-210, 1990

2. Vinée P, Tanyü MO, Hauenstein KH, Sigmund G, Stöver B and Adler CP: CT and MRI of Gorham syndrome. J Comput Assist Tomogr 18: 985-989, 1994.

3. Tie ML, Poland GA and Rosenow EC III: Chylothorax in Gorham's syndrome. A common complication of a rare disease. Chest 105: 208-213, 1994.

4. Mawk JR, Obukhov SK, Nichols WD, Wynne TD, Odell JM and Urman SM: Successful conservative management of Gorham disease of the skull base and cervical spine. Childs Nerv Syst 13: 622-625, 1997.

5. Schell A, Rhee JM, Allen A, Andras L and Zhou F: Surgical management of Gorham disease involving the upper cervical spine with occipito-cervical-thoracic fusion: A case report. Spine J 16: e467-e472, 2016.

6. Gorham LW and Stout AP: Massive osteolysis (acute spontaneous absorption of bone, phantom bone, disappearing bone); its relation to hemangiomatosis. J Bone Joint Surg Am 37-A 985-1004, 1955.
7. Hellyer J, Oliver-Allen H, Shafiq M, Tolani A, Druzin M, Jeng M, Rockson S and Lowsky R: Pregnancy complicated by gorham-stout disease and refractory chylothorax. AJP Rep 6: e355-e358, 2016

8. Möller G, Priemel M, Amling M, Werner M, Kuhlmey AS and Delling G: The Gorham-Stout syndrome (Gorham's massive osteolysis). A report of six cases with histopathological findings. J Bone Joint Surg Br 81: 501-506, 1999.

9. Heyden G, Kindblom LG and Nielsen JM: Disappearing bone disease. A clinical and histological study. J Bone Joint Surg Am 59: 57-61, 1977.

10. Hammer F, Kenn W, Wesselmann U, Hofbauer LC, Delling G, Allolio B and Arlt W: Gorham-Stout disease: Stabilization during bisphosphonate treatment. J Bone Miner Res 20: 350-353, 2005.

11. Hominick D, Silva A, Khurana N, Liu Y, Dechow PC, Feng JQ, Pytowski B, Rutkowski JM, Alitalo K and Dellinger MT: VEGF-C promotes the development of lymphatics in bone and bone loss. Elife 7: e34323, 2018.

12. Baud J, Lomri A, Graber D and Bikfalvi A: The therapeutic response in Gorham's syndrome to the beta-blocking agent propranolol is correlated to VEGF-A, but not to VEGF-C or FLT1 expression. BMC Res Notes 8: 333, 2015.

13. Heffez L, Doku HC, Carter BL and Feeney JE: Perspectives on massive osteolysis. Report of a case and review of the literature. Oral Surg Oral Med Oral Pathol 55: 331-343, 1983.

14. Kim JH, Yoon DH, Kim KN, Shin DA, Yi S, Kang J and Ha Y: Surgical management of gorham-stout disease in cervical compression fracture with cervicothoracic fusion: Case report and review of literature. World Neurosurg 129: 227-281, 2019.

15. Tena-Sanabria ME, Jesús-Mejenes LY, Fuentes-Herrera G, Álvarez-Martínez FA, Victorio-García NP and NúñezEnríquez JC: A report of two children with Gorham-Stout disease. BMC Pediatr 19: 206, 2019.

16. Tateda S, Aizawa T, Hashimoto K, Kanno H, Ohtsu S, Itoi E and Ozawa H: Successful management of gorham-stout disease in the cervical spine bycombined conservative and surgical treatments: A Case Report. Tohoku J Exp Med 241: 249-254, 2017.

17. Noda M, Endo C, Hoshikawa Y, Ishibashi N, Suzuki T, Okada Y and Kondo T: Successful management of intractable chylothorax in Gorham-Stout disease by awake thoracoscopic surgery. Gen Thorac Cardiovasc Surg 61: 356-358, 2013.

18. Heyd R, Micke O, Surholt C, Berger B, Martini C, Füller J, Schimpke T and Seegenschmiedt MH; German Cooperative Group on Radiotherapy for Benign Diseases (GCG-BD): Radiation therapy for Gorham-Stout syndrome: Results of a national patterns-of-care study and literature review. Int J Radiat Oncol Biol Phys 81: e179-e185, 2011.

19. Kai B, Ryan A, Munk PL and Dunlop P: Gorham disease of bone: Three cases and review of radiological features. Clin Radiol 61: 1058-1064, 2006

20. Lekovic GP, Mariwalla NR, Horn EM, Chang S, Rekate HL and Theodore N: Skeletal dysplasia involving the subaxial cervical spine. Report of two cases and review of the literature. Neurosurg Focus 20: E8, 2006.

21. Chong Ng L and Sell P: Gorham disease of the cervical spine-a case report and review of the literature. Spine 28: E355-E358, 2003.

22. Bode-Lesniewska B, von Hochstetter A, Exner GU and Hodler J: Gorham-Stout disease of the shoulder girdle and cervico-thoracic spine: Fatal course in a 65-year-old woman. Skeletal Radiol 31: 724-729, 2002.

23. Chung C, Yu JS, Resnick D, Vaughan LM and Haghighi P: Gorham syndrome of the thorax and cervical spine: CT and MRI findings. Skeletal Radiol 26: 55-59, 1997.

24. Hagberg H, Lamberg K and Aström G: Alpha-2b interferon and oral clodronate for Gorham's disease. Lancet 350: 1822-1823, 1997.

25. Khosrovi H, Ortiz O, Kaufman HH, Schochet SS Jr, Reddy GN and Simmons D: Massive osteolysis of the skull and upper cervical spine. Case report and review of the literature. J Neurosurg 87: 773-780, 1997.

26. Liu Y, Zhong DR, Zhou PR, Lv F, Ma DD, Xia WB, Jiang Y, Wang O, Xing XP and Li M: Gorham-Stout disease: Radiological, histological, and clinical features of 12 cases and review of literature. Clin Rheumatol 35: 813-823, 2016.

27. Chavanis N, Chaffanjon P, Frey G, Vottero G and Brichon PY: Chylothorax complicating Gorham's disease. Ann Thorac Surg 72: 937-939, 2001.

28. Brodszki N, Länsberg JK, Dictor M, Gyllstedt E, Ewers SB, Larsson MK and Eklund EA: A novel treatment approach for paediatric Gorham-Stout syndrome with chylothorax. Acta Paediatr 100: 1448-1453, 2011. 